\title{
Analysis of Wavelet Denoising of a Colour ImageWith Different Types of Noises
}

\author{
Prateek Kumar and Sandeep Kumar Agarwal \\ Department of Electronics Communication Engineering \\ RustamJi Institute of Technology, BSF Academy, Tekanpur, Gwalior (M.P.)- \\ INDIA \\ kumarprateek34@gmail.com, rjitsandeep@gmail.com
}

\begin{abstract}
There are various types of noises that affect quality of an image such as Salt-andpepper noise, Poison noise, Gaussian noise, Speckle noise etc. Wavelet is a powerful tool for denoising a variety of signals. Here a White Flower image has been taken for denoising purpose with the help of HAAR Transform. The noisy image is first decomposed into five levels to obtain different frequency bands. Then hard thresholding method is used to remove the noisy coefficients by fixing the optimum thresholding value. In this paper, analysis of a colored image is carried out with four different noises at zero mean that are applied on the image to produce noisy images. Residual image is obtained from the original and noisy image \& its statistical parameters such as mean, median, mode, standard deviation, mean absolute deviation, median absolute deviation are calculated. In order to enhance the quality of the noisy images, performance parameters of denoised images must be estimated. The comparison between noisy and denoised image is taken in terms of MSE (mean square error), PSNR (peak signal to noise ratio), RMSE (root mean square error), SNR (signal to noise ratio) and SSIM (structural similarity index).
\end{abstract}

Keywords: salt-and-pepper noise; poison noise;gaussian noise; speckle noise; Wavelet transform;hard thresholding ; psnr; snr; rmse; mse; ssim

\section{Introduction}

An image is often corrupted by noise during its acquisition or transmission. The denoising process is to remove the noise while retaining and not distorting the quality of processed image. Denoising analysis of the images is done by using HAAR Wavelet Transform. The experiments are conducted on a white color flower image (.jpg format).

Simple denoising algorithms that used DWT consist of three steps [1]:

1. Discrete wavelet transform is adopted to decompose the noisy image and get the wavelet coefficients.

2. These wavelet coefficients are denoised with wavelet threshold.

3. Inverse transform is applied to the modified coefficients and get denoised image.

Thresholding is a simple non-linear technique which operates on one of the wavelet coefficients at a time. Each coefficient is threshold by comparing threshold level. When a coefficient is smaller than threshold level than it is set to zero otherwise it is kept as it is or it is modified [1]. 


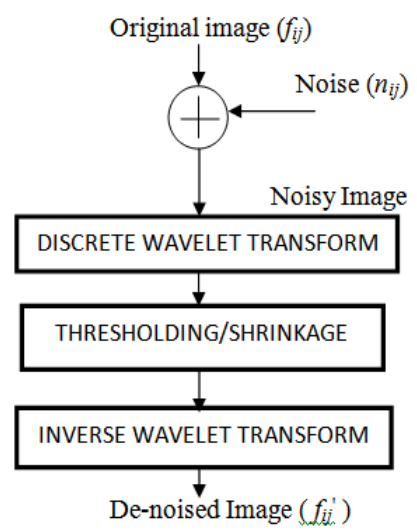

Figure 1. The Frame Work of Image Denoising based on Wavelet Transforms [1]

The wavelet transform is better than Fourier transform because it gives frequency representation of raw signal at any given interval of time, but Fourier transform gives only the frequency- amplitude representation of the raw signal but the time information is lost. So we cannot use the Fourier transform where we need time as well as frequency information at particular time. The fundamental idea behind wavelets is to analyze the signal at different scales or resolutions, which is called multiresolution. The most important feature of wavelet transform is it allows multiresolution decomposition [3]. The wavelet transform is good at energy compaction. The small coefficients are more likely due to noise and large coefficient are more likely due to important signal features. These small coefficients can be threshold without affecting the significant features of the image.

Noise is a random variation of image Intensity and visible as grains in the image. It may arise in the image as effects of basic physics-like photon nature of light or thermal energy of heat inside the image sensors [14].

Here we are discussing about four types of noise and their effect on the image signal.

1. Gaussian noise

2. Speckle noise

3. Salt-and-pepper noise

4. Poison noise

Gaussian noise is statistical noise having a probability density function (PDF) equal to that of the normal distribution, which is also known as the Gaussian distribution[6].This noise model is additive in nature [8].Additive white Gaussian noise (AWGN) can be caused by poor quality image acquisition, noisy environment or internal noise in communication channels.

Speckle-noise is a granular noise degrades the quality of the active radar, synthetic aperture radar (SAR), and medical ultrasound images. Speckle noise occurs in conventional radar due to random fluctuations in the return signal from an object [9].

Salt-and-pepper noise is also called impulsive noise or spike noise [6]. Salt-and-pepper noised image has dark pixels in bright area and bright pixels in dark area of the image. It has only two possible values, a high value and a low value. This noise occurs during analog-to-digital converter errors, bit errors in transmission [9].

Poisson noise or Shot noise is an electronic noise which is modeled by a Poisson process. Shot noise is related to the particle nature of light. Poisson or shot photon noise is the noise that occurs when a number of photons sensed by the sensor is not sufficient to provide detectable statistical information. This noise has root mean square value proportional to square root intensity of the image [8]. 


\section{Discrete Wavelet Transform}

In Discrete Wavelet Transform signal energy is concentrated in a small number of coefficients. DWT of noisy image consist of small number of coefficients having high SNR and large number of coefficients having low SNR. Using inverse DWT, image is reconstructed after removing the coefficients with low SNR. Time and frequency localization is simultaneously provided by Wavelet transform. When DWT is applied to noisy image, it is divided into four sub bands as shown in Figure 1(a).These sub bands are formed by separable applications of horizontal and vertical filters. Coefficients that are represented as sub bands LH1, HL1 and HH1 are detail images while coefficients are represented as sub band LL1 is approximation image [15]. The LL1 sub band is further decomposed to obtain the next level of wavelet coefficients as shown in Figure 1(b).
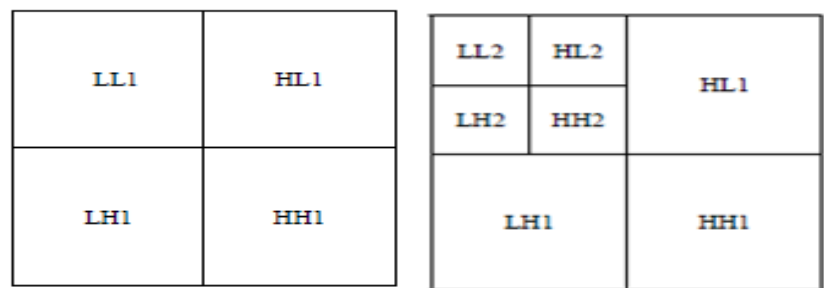

(a) One- Level (b) Two- Level

\section{Figure 2. Image Decomposition by using DWT [15]}

LL1 is called the approximation sub band as it provides the most like original picture. It comes from low pass filtering in both directions. The other bands are called detail sub bands. The filters $\mathrm{L}$ and $\mathrm{H}$ as shown in Figure 2 are one dimensional low pass filter (LPF) and high pass filter (HPF) for image decomposition. HL1 is called the horizontal fluctuation as it comes from low pass filtering in vertical direction and high pass filtering in horizontal direction. LH1 is called vertical fluctuation as it comes from high pass filtering in vertical direction and low pass filtering in horizontal direction. HH1 is called diagonal fluctuation as it comes from high pass filtering in both the directions. LL1 is decomposed into 4 sub bands LL2, LH2, HL2 and HH2. The process is carried until the fifth decomposition is reached. After $\mathrm{L}$ decompositions a total of $\mathrm{D}(\mathrm{L})=3 * \mathrm{~L}+1$ sub bands are obtained. Therefore after 5 decompositions $\mathrm{D}(5)=3 * 5+1=16$ sub bands are obtained. The decomposed image can be reconstructed by reverse process as shown in Figure 3. Here, the filters $\mathrm{L}$ and $\mathrm{H}$ represent low pass and high pass reconstruction filters respectively.

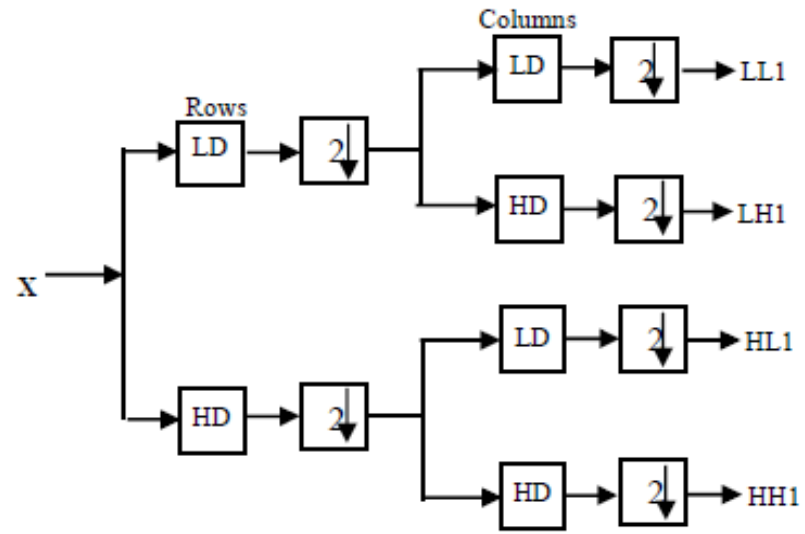

Figure 3. Wavelet Filter Bank for One-level Image Decomposition [15] 


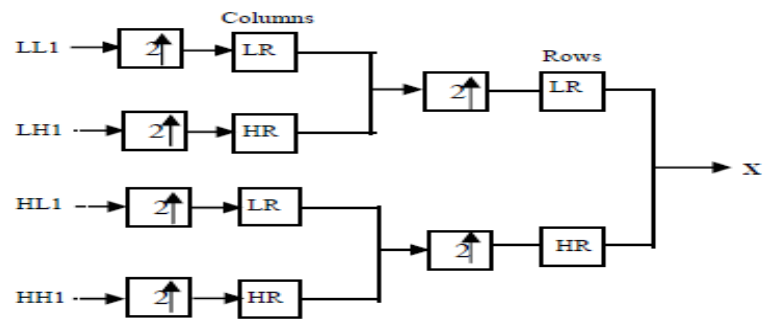

Figure 4. Wavelet Filter Bank for One-level Image Reconstruction [15]

\section{Wavelet Thresholding}

Thresholding technique operates on one of the wavelet coefficients at a time. In it the coefficient which is smaller than threshold is set to zero otherwise it is kept or modified [3].

The small valued coefficients carry more noise than large valued coefficients while large valued coefficients carry more signal information than small valued coefficients. So noise coefficients or small coefficients below a certain threshold value are replaced by zero and an inverse wavelet transform may lead to a reconstruction that has lesser noise [5].

Firstly wavelet analysis of a noisy image up to level $\mathrm{N}$ is done Secondly thresholding of the each detail coefficients from level 1 to $\mathrm{N}$ is done. Lastly signal is synthesized by using the altered detail coefficients from level 1 to $\mathrm{N}$ and approximation coefficients of level $\mathrm{N}$.

There are two types of thresholding;

1. Hard thresholding technique

2. Soft thresholding technique

Hard thresholding shrinks the coefficients which have magnitudes below the threshold level, and leaves the rest of the coefficients unchanged.

To suppress the noise we apply hard thresholding on each wavelet coefficients:

$\mathrm{F}(\mathrm{x})=\mathrm{x} \times \mathrm{I}(|\mathrm{x}|>t)$

Here ' $\mathrm{t}$ ' is a certain threshold and $\mathrm{x}$ is the coefficient.

$\mathrm{t}=\sqrt{\left(\frac{2 \sigma^{2} \log (\mathrm{n})}{\mathrm{n}}\right)}$

Where ' $n$ ' is the length of the input vector and $\sigma^{2}$ is the variance of the noise.

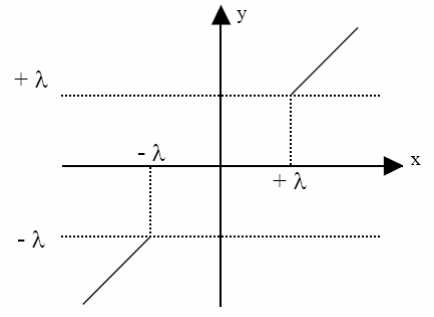

Figure 5. Hard Thresholding [14]

Soft thresholding technique extends hard thresholding technique by shrinking the magnitude of the remaining coefficients by $\mathrm{T}$ factor and producing a smooth rather than abrupt transition to zero [14].

Soft thresholding nonlinear transform is used

$\mathrm{S}(\mathrm{x})=\operatorname{sign}(\mathrm{x})(|\mathrm{x}|-\mathrm{t}) \mathrm{I}(|\mathrm{x}|>t)$ 


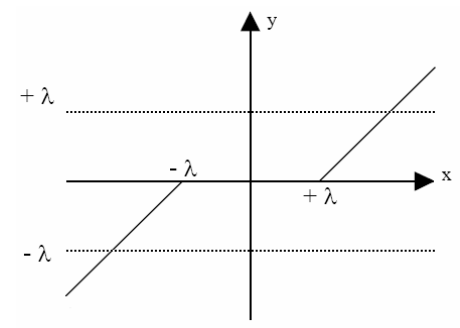

Figure 6. Soft Thresholding [14]

\section{Performance Parameters}

For comparing original white color image with noisy and denoised images, we calculate following parameters:

1. Mean Square Error (MSE): The MSE is the cumulative square error between the synthesized image and the original image defined by:

$$
\operatorname{MSE}=\sum_{0}^{\mathrm{m}-1} \sum_{0}^{\mathrm{n}-1}|| \mathrm{f}(\mathrm{i}, \mathrm{j})-\left.\mathrm{g}(\mathrm{i}, \mathrm{j})\right|^{2}
$$

Where ' $\mathrm{f}$ ' is the original image and ' $\mathrm{g}$ ' is the synthesized image. MSE should be as low as possible.

2. Peak signal to Noise ratio (PSNR): PSNR is the ratio between maximum possible power of a signal and the power of distorting noise which affects the quality of the original signal. It is defined by:

$$
\text { PSNR }=\frac{20 \log _{10}\left(\text { MAX }_{\mathrm{F}}\right)}{\sqrt{\mathrm{MSE}}}
$$

Where $\mathrm{MAX}_{\mathrm{F}}$ is the maximum signal value that exists in our original image. PSNR should be as high as possible.

3. Root mean square error (RMSE): It measures of the differences between value predicted by a model or an estimator and the values actually observed [12]. It is Possible. the square root of mean square error. RMSE should be as low as

$\operatorname{RMSE}=\sqrt{\mathrm{MSE}}[12]$

4. Structural Similarity Index (SSIM): It is a method for measuring the similarity between two images. The SSIM measure the image quality based on an initial distortion-free image as reference.

$\mu_{\mathrm{X}}$ is average of $\mathrm{x}$;

$$
\operatorname{SSIM}=\frac{\left(2 \mu_{\mathrm{X}} \mu_{\mathrm{Y}}+\mathrm{C}_{1}\right)\left(2 \sigma_{\mathrm{XY}} \mathrm{C}_{2}\right)}{\left(\mu_{\mathrm{X}}^{2}+\mu_{\mathrm{Y}}^{2}+\mathrm{C}_{1}\right)\left(\sigma_{\mathrm{X}}^{2}+\sigma_{\mathrm{Y}}^{2}+\mathrm{C}_{2}\right)}
$$

$\mu_{Y}$ is average of $y$;

$\sigma_{\mathrm{X}}^{2}$ is variance of $\mathrm{x}$;

$\sigma_{\mathrm{Y}}^{2}$ is variance of $\mathrm{y}$;

$\sigma_{X Y}$ is covariance of $\mathrm{x}$ and $\mathrm{y}$;

$C_{1}=\left(\mathrm{k}_{1} \mathrm{~L}\right)^{2}$ and $C_{2}=\left(\mathrm{k}_{2} \mathrm{~L}\right)^{2}$ are two variables to stabilize the division with weak denominator. $\mathrm{L}$ the dynamic range of the pixel-values $\mathrm{k}_{1}=0.01$ and $\mathrm{k}_{2}=0.03$ by default.

5. Signal to noise ratio (SNR): Signal-to-noise ratio is defined as the power ratio between a signal (meaningful information) and the noise (unwanted signal).It should be as low as possible.

$S N R=\frac{P_{\text {SIGNAL }}}{P_{\text {NOISE }}}[13]$ 


\section{Statistical Parameters}

1. Mean: It is the average value of a sample of a data set and is computed as the sum of all the observed outcomes from the sample divided by the total number of events.

$x=\frac{1}{n} \sum_{i=1}^{n} x$

Here $\mathrm{n}=$ sample size $\& \mathrm{x}=$ observed valued.

2. Median: It is the middle term of the set of data.

3. Mode: The mode is a set of data is the number which has highest frequency.

4. Standard Deviation: It is the square root of the variance.

$S=\sqrt{\frac{1}{n-1} \sum_{i=1}^{n}(x-\bar{x})^{2}}$

5. Median Absolute Deviation (MAD): It is a measure of variability of a sample of quantitative data. A sample of data set $\mathrm{X}_{1}, \mathrm{X}_{2} \ldots \mathrm{Xi}$

$$
M A D=\operatorname{median}_{i}\left(\left|X_{I}-\operatorname{median}_{i}\left(X_{i}\right)\right|\right)
$$

6. Mean Absolute Deviation or Error: It is a quantity used to measure closeness of predictions to the true value.

$M A E=\frac{1}{n} \sum_{i=1}^{n}\left|f_{i}-y_{i}\right|=\frac{1}{n} \sum_{i=1}^{n}\left|e_{i}\right|$

It is an average of absolute errors ' $\mathrm{e}_{\mathrm{i}}$ '. ' $\mathrm{f}_{\mathrm{i}}$ ' is the prediction and ' $\mathrm{y}_{\mathrm{i}}$ ' is the true value.

\section{Methodology}

1. A White-Flower image has been taken for denoising purpose in Wavelet Toolbox in MATLAB. Four different noises (Gaussian, Poisson, Speckle, Salt and pepper) are added one by one in the original image of dimensions 832x640 in jpg format at zero mean and 0.02 variance. HAAR wavelet transform is applied on the noisy images of all the four different noises to decompose the noisy image up to five levels. A constant threshold value is taken at each level. Each coefficient of vertical, horizontal and diagonal details is thresholded using hard threshold. As a result of inverse wavelet transform, we get the noise free image within vertical, horizontal and diagonal details.

2. Calculation of the statistical parameters for residual image is obtained in the terms such as: (a) mean (b) median (c) mode (d) standard deviation (e) mean absolute deviation and (f) median absolute deviation.

3. Calculation of the performance parameters for analyzing noisy images and denoised images is performed in the terms such as: (a) RMSE (b) SNR (c) PSNR (d) MSE and (e) SSIM.

\section{Result}

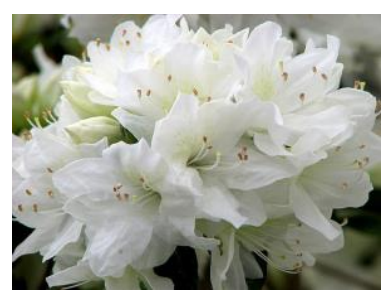

(a)

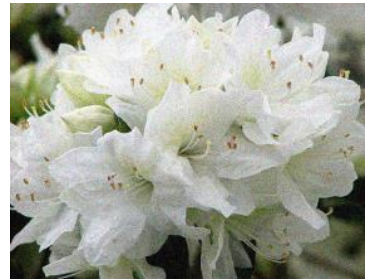

(b)

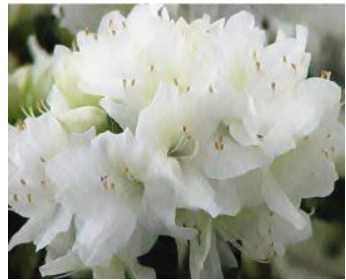

(c) 


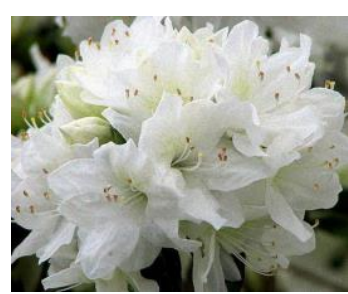

(d)

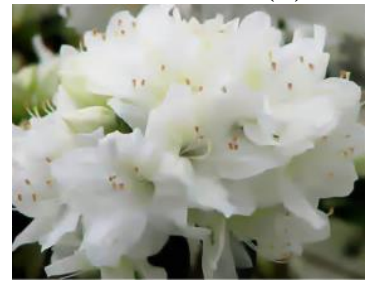

(g)

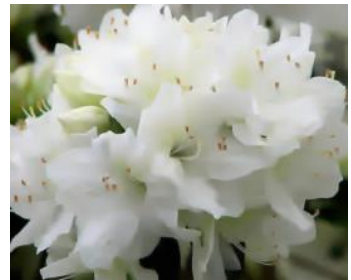

(e)

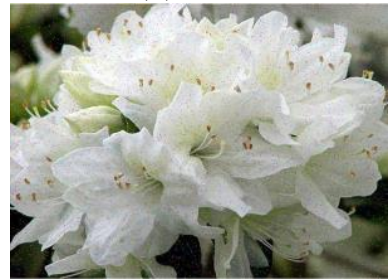

(h)

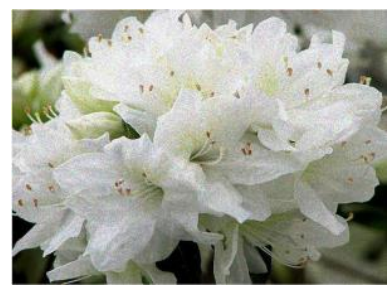

(f)

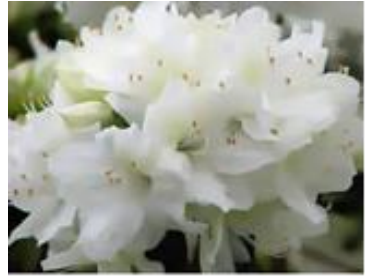

(i)

Figure 7. (a) Original White color flower, (b) Image obtained after adding Gaussian noise, (c) Image obtained after denoising Gaussian noise, (d)

Image obtained after adding Poison noise, (e) Image obtained after denoising poison noise, (f) Image obtained after adding Speckle noise, (g) Image obtained after denoising Speckle noise, (h) Image obtained after adding Salt and Pepper noise, (i) Image obtained after denoising Salt and Pepper noise

Table 1. Noises at Zero Mean And At .02 Variance-And Parameters Of Residual Image

\begin{tabular}{|l|l|l|l|l|l|l|}
\hline \multirow{2}{*}{$\begin{array}{c}\text { Noises } \\
\text { At Zero } \\
\text { Mean } \\
\begin{array}{c}\text { And At } \\
\text {.02 } \\
\text { Variance }\end{array}\end{array}$} & $\begin{array}{l}\text { mea } \\
n\end{array}$ & $\begin{array}{c}\text { medi } \\
\text { an }\end{array}$ & $\begin{array}{c}\text { mod } \\
\text { e }\end{array}$ & $\begin{array}{l}\text { Std. } \\
\text { dev. }\end{array}$ & $\begin{array}{c}\text { Mea } \\
\boldsymbol{n} \\
\text { abs. } \\
\text { dev. }\end{array}$ & $\begin{array}{c}\text { Medi } \\
\text { an } \\
\text { abs. } \\
\text { dev. }\end{array}$ \\
\hline $\begin{array}{l}\text { Salt and } \\
\text { Pepper }\end{array}$ & $\begin{array}{l}- \\
0.01 \\
1\end{array}$ & 0 & 1.29 & $\begin{array}{l}11.1 \\
5\end{array}$ & 6.05 & 3 \\
\hline Poisson & - & 0 & - & 10.9 & 9.83 & 4 \\
& 0.01 & & 0.91 & 4 & 9 & \\
\hline Gaussian & - & 0 & 1 & 13.0 & $\begin{array}{l}8.36 \\
9\end{array}$ & 5 \\
& 0.12 & & & 4 & 9 & \\
\hline Speckle & - & 0 & 0.52 & 12.4 & 7.77 & 4 \\
& 0.01 & & & 2 & 7 & \\
\hline
\end{tabular}

Table 2. Noises at Zero Mean And 0.02 Variance And PSNR

\begin{tabular}{|c|c|c|c|}
\hline \multirow{2}{*}{$\begin{array}{c}\text { Noises At } \\
\text { zero mean } \\
\text { and 0.02 } \\
\text { variance }\end{array}$} & $\begin{array}{c}\text { PNR of } \\
\text { noisy } \\
\text { image }\end{array}$ & $\begin{array}{c}\text { PSNR of } \\
\text { denoised } \\
\text { image }\end{array}$ & $\begin{array}{c}\text { rcentage rise } \\
\text { in PSNR }\end{array}$ \\
\hline
\end{tabular}




\begin{tabular}{|l|l|l|l|}
\hline $\begin{array}{l}\text { Salt and } \\
\text { Pepper }\end{array}$ & 27.4852 & 31.4783 & $14.5282 \%$ \\
\hline Poison & 27.8487 & 31.7198 & $13.9004 \%$ \\
\hline Gaussian & 25.5083 & 30.0746 & $17.9010 \%$ \\
\hline Speckle & 25.9409 & 30.7518 & $18.5457 \%$ \\
\hline
\end{tabular}

Table 3. Noises at Zero Mean and 0.02 Variance And SSIM

\begin{tabular}{|l|l|l|l|}
\hline \multirow{2}{*}{$\begin{array}{c}\text { Noises At } \\
\text { Zero } \\
\begin{array}{c}\text { Mean And } \\
\mathbf{0 . 0 2} \\
\text { Variance }\end{array}\end{array}$} & $\begin{array}{c}\text { SSIM } \\
\text { of noisy } \\
\text { image }\end{array}$ & $\begin{array}{c}\text { SSIM of } \\
\text { denoised } \\
\text { image }\end{array}$ & $\begin{array}{c}\text { Percentage } \\
\text { increase in } \\
\text { SSIM }\end{array}$ \\
\hline $\begin{array}{l}\text { Salt and } \\
\text { Pepper }\end{array}$ & 0.7607 & 0.8946 & $17.5873 \%$ \\
\hline Poison & 0.7285 & 0.9041 & $24.1028 \%$ \\
\hline Gaussian & 0.6543 & 0.8870 & $35.5260 \%$ \\
\hline Speckle & 0.6843 & 0.8982 & $31.2581 \%$ \\
\hline
\end{tabular}

Table 4. Noises at Zero Mean And 0.02 Variances

\begin{tabular}{|l|l|l|l|}
\hline \multirow{2}{*}{$\begin{array}{c}\text { Noises at } \\
\text { zero mean } \\
\text { and 0.02 } \\
\text { variance }\end{array}$} & $\begin{array}{c}|c| \\
\text { MSE of } \\
\text { noisy } \\
\text { image }\end{array}$ & $\begin{array}{c}\text { MSE of } \\
\text { denoised } \\
\text { image }\end{array}$ & $\begin{array}{c}\text { Percentag } \\
\text { e reduce in } \\
\text { MSE }\end{array}$ \\
\hline $\begin{array}{l}\text { Salt and } \\
\text { pepper }\end{array}$ & $\begin{array}{l}5.2665 \mathrm{e} \\
+05\end{array}$ & $4.6435 \mathrm{e}+05$ & $11.8295 \%$ \\
\hline Poisson & $\begin{array}{l}5.1989 \mathrm{e} \\
+05\end{array}$ & $4.6127 \mathrm{e}+05$ & $11.2750 \%$ \\
\hline Gaussian & $\begin{array}{l}5.6863 \mathrm{e} \\
+05\end{array}$ & $4.8359 \mathrm{e}+05$ & $14.9555 \%$ \\
\hline Speckle & $\begin{array}{l}5.5860 \mathrm{e} \\
+05\end{array}$ & $4.7400 \mathrm{e}+05$ & $15.1445 \%$ \\
\hline
\end{tabular}

Table 5. Noises at Zero Mean And 0.02 Variance and SNR

\begin{tabular}{|l|l|l|l|}
\hline $\begin{array}{c}\text { Noises At } \\
\text { Zero } \\
\begin{array}{c}\text { Mean And } \\
\text { 0.02 } \\
\text { Variance }\end{array}\end{array}$ & $\begin{array}{c}\text { SNR of } \\
\text { noisy } \\
\text { image }\end{array}$ & $\begin{array}{c}\text { SNR of } \\
\text { denoised } \\
\text { image }\end{array}$ & $\begin{array}{c}\text { Percentage } \\
\text { increase in } \\
\text { SNR }\end{array}$ \\
\hline $\begin{array}{l}\text { Salt and } \\
\text { pepper }\end{array}$ & 25.4981 & 29.4912 & $15.6605 \%$ \\
\hline Poisson & 25.8612 & 29.7327 & $14.9685 \%$ \\
\hline Gaussian & 23.5212 & 28.0875 & $19.4133 \%$ \\
\hline Speckle & 23.9569 & 28.7678 & $20.0815 \%$ \\
\hline
\end{tabular}

Table 6. Noises at Zero Mean and 0.02 Variance And RMSE

\begin{tabular}{|c|c|c|c|}
\hline \multirow{2}{*}{$\begin{array}{c}\text { Noises At } \\
\text { Zero } \\
\text { Mean And } \\
0.02\end{array}$} & \multicolumn{3}{|c|}{ RMSE } \\
\hline & $\begin{array}{c}\text { RMSE of } \\
\text { noisy image }\end{array}$ & $\begin{array}{c}\text { RMSE of } \\
\text { denoised } \\
\text { image }\end{array}$ & $\begin{array}{c}\text { Percentage } \\
\text { reduce in } \\
\text { RMSE }\end{array}$ \\
\hline
\end{tabular}




\begin{tabular}{|l|l|l|l|}
\hline \multicolumn{1}{|c|}{ Variance } & & & \\
\hline $\begin{array}{l}\text { Salt-and- } \\
\text { pepper }\end{array}$ & $7.2570 \mathrm{e}+02$ & $\begin{array}{l}6.8143 \mathrm{e}+0 \\
2\end{array}$ & $6.1008 \%$ \\
\hline Poisson & $7.2103 \mathrm{e}+02$ & $\begin{array}{l}6.7917 \mathrm{e}+0 \\
2\end{array}$ & $5.8060 \%$ \\
\hline Gaussian & $7.5407 \mathrm{e}+02$ & $\begin{array}{l}6.9540 \mathrm{e}+0 \\
2\end{array}$ & $7.7804 \%$ \\
\hline Speckle & $7.4739 \mathrm{e}+02$ & $\begin{array}{l}6.8847 \mathrm{e}+0 \\
2\end{array}$ & $7.8829 \%$ \\
\hline
\end{tabular}

\section{Conclusion}

In this paper:

1. Speckle denoised image has the highest Percentage (18.54\%) increase in PSNR in comparison to other denoised images of the different noises under same condition.

2. Gaussian denoised image has the highest Percentage (35.52\%) increase in SSIM in comparison to other denoised images of the different noises under same condition.

3. Speckle denoised image has the highest Percentage (20.08\%) increase in SNR in comparison to other denoised images of the different noises under same condition 4. Speckle denoised image has the highest Percentage (15.14\%) decrease in MSE in comparison to other denoised images of the different noises under same condition 5. Speckle denoised image has the highest Percentage (7.88\%) decrease in RMSE in comparison to other denoised images of the different noises under same condition 6. Speckle denoised image has better results during PSNR, SNR, RMSE, MSE calculation under same conditions as in other four noises. We generate MATLAB codes for all the operations like -Noising, Denoising, PSNR, SNR, SSIM, MSE and RMSE.

7. Here, it is concluded that HAAR wavelet transform provides better results for denoising speckle noise from an image.

\section{Future Work}

In future work;

1. Here we have gone through level 5 decomposition and we can make it to higher level decomposition up to 8 levels to be more accurate and clear.

2. Here we use Salt-and-pepper noise, Poison noise, Gaussian noise and speckle noise to perform experiments in future we may use different types of noises also.

3. We may calculate more different statistics as well as performance parameters such as L2NORM, L1 NORM, EME, PCC etc.

\section{References}

[1] V. Mahesh, M. Someswara Rao, Ch. Sravani, P. Durgarao and S. Venkatesh, "An Effective Image Denoising Using Adaptive Thresholding In Wavelet Domain",Int. Journal of Engineering Research and Applications www.ijera.com ISSN : 2248-9622, vol. 4, no. 4 (Version 1), (2014) April, pp. 365-368.

[2] A. K. Das, "Review on Image Denoising Techniques", International Journal of Emerging Technology and Advanced Engineering Website: www.ijetae.com (ISSN 2250-2459, ISO 9001:2008 Certified Journal, Volume 4, Issue 8, August 2014). 2248-9622, vol. 4, no. 4 (Version 1), (2014), April, pp. 365368.

[3] V. Sharan, N. Keshari and T. Mondal, "Biomedical Image Denoising and Compression in Wavelet using MATLAB", International Journal of Innovative Science and Modern Engineering (IJISME) ISSN: 23196386, vol. 2, no. 6, (2014), May.

[4] A. Vishwa and V. Goyal, "An Improved Threshold Estimation Technique for Ultrasound Image Denoising", International Journal of Advanced Research in Computer Science and Software Engineering Research Paper, vol. 3, no. 7, July 2013 ISSN: 2277 128X Available online at: www.ijarcsse.com.

[5] A. A. Jumah, "Denoising of an Image Using Discrete Stationary WaveletTransform and Various Thresholding Techniques", Journal of Signal and Information Processing, (2013, 4, 33-41 
http://dx.doi.org/10.4236/jsip.2013.41004 Published Online February 2013 (http://www.scirp.org/journal/jsip).

[6] P. Kamboj and V. Rani, "A BRIEF STUDY OF VARIOUS NOISE MODEL AND FILTERING TECHNIQUES", Journal of Global Research in Computer Science REVIEW ARTICLE Available Online at www.jgrcs.info $\odot$, vol. 4, no. 4, (2013) April.

[7] A. Jaiswal, J. P. Upadhyay, R. Mohan and S. P. Bohre, "A Novel Approach for Reduction of Poisson Noise in Digital Images”, ISSN : 2248-9622, vol. 3, no. 5, (2013), September-October, pp. 1275-1279.

[8] R. Verma and J. Ali "A Comparative Study of Various Types of Image Noise and Efficient Noise Removal Techniques", International Journal of Advanced Research in Computer Science and Software Engineering, vol. 3, no. 10, (2013) October, ISSN: 2277 128X.

[9] Anutam and Rajni, "COMPARATIVE ANALYSIS OF FILTERSAND WAVELET BASED THRESHOLDINGMETHODS FOR IMAGE DENOISING", International Journal of Computer Applications (0975 - 8887) vol. 86, no. 16, (2012) January.

[10] I. M. G. Alwan, "Color Image Denoising Using Stationary Wavelet Transform and Adaptive Wiener Filter", Al-Khwarizmi Engineering Journal, vol. 8, no. 1, (2012), pp. 18 -26.

[11] Y. A. Y. Al-Najjar and D. C. Soong, "Comparison of Image Quality Assessment: PSNR, HVS, SSIM, UIQI”, International Journal of Scientific \& Engineering Research, vol. 3, no. 8, (2012 ), August ,1 ISSN 2229-5518

[12] A. Saffor, A. R. Ramli and K.-H. Ng, “A COMPARATIVE STUDY OF IMAGE COMPRESSION BETWEEN JPEG AND WAVELET", Malaysian Journal of Computer Science, vol. 14, no. 1, June 2011, pp. 39-45.

[13] S. D. Ruikar and D. D. Doye, "Wavelet Based Image Denoising Technique", (IJACSA) International Journal of Advanced Computer Science and Applications, vol. 2, no. 3, (2011) March.

[14] M. Mastriani, 'Denoising and Compression in Wavelet Domain via Projection onto Approximation Coefficients", World Academy of Science, Engineering and Technology, vol. 3, (2009), pp. 11-20.

[15] D. Gnanadurai and V. Sadasivam, "An Efficient Adaptive Thresholding Technique for Wavelet Based Image Denoising", World Academy of Science, Engineering and Technology, vol. 2, (2008), pp. 8-20.

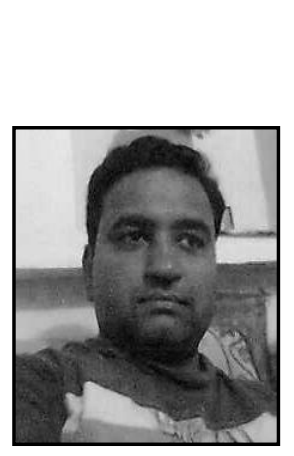

\section{Author}

Sandeep Kumar Agrawal, he received his Bachelor's Degree in Electronics Engineering in 2002 from the Rajiv Gandhi Technical University Madhya Pradesh, India and M.Tech. Degree in Electronics \& Communication with specialization in Microwave Engineering from Madhav Institute of technology \& Science, Gwalior, India in 2005. He is currently working as an Assistant Professor of Electronics Engg in Rustamji Institute of Technology, BSF Academy, Tekanpur, Gwalior (India). His research work area includes image compression, image denoising \& image fusion. 